\title{
Perspektiven der Transduceroptimierung - Einfluss des Elektrodenmaterials bei verschiedenen Temperaturen auf $\mathrm{SnO}_{2}$ Dickschicht Sensoren
}

\author{
Sven Rank, Geoffroy Gadacz, Nicolae Barsan, Udo Weimar \\ Institut für Physikalische und Theoretische Chemie, Universität Tübingen, Auf der Morgenstelle 15, \\ 72076 Tübingen
}

\section{Zusammenfassung}

In der vorliegenden Arbeit wurden $\mathrm{SnO}_{2}$ Dickschicht Sensoren mit unterschiedlichem Elektrodenmaterial bei verschiedenen Arbeitstemperaturen in Bezug auf ihre Leistungscharakteristik untersucht. Während bekannte Eigenschaften bezüglich einer optimierten Sensitivität für Kohlenmonoxid und Wasserstoff mit Gold oder Platin als Elektrodenmaterial bestätigt werden konnten [1], wurde ein weiterer Ausblick auf die Stabilität sowie Sensitivität der Sensoren bei unterschiedlichen Temperaturen bezüglich der untersuchten Gase gegeben. Mit dieser Arbeit konnte eine statistische Grundlage für weitere Arbeiten bezüglich der Aufklärung des Einflusses von verschiedenen Elektrodenmaterialen gegeben werden.

\section{Einführung}

Der Einfluss des Elektrodenmaterials sowie der geometrische Aufbau der Elektrode bei Metalloxid Gas Sensoren wurde in zahlreichen Publikationen behandelt [2,3]. Dennoch gibt es immer noch offene Fragen für das tiefere Verständnis dieser Beeinflussung, welches fundamental für eine weitere Verbesserung der Leistung dieser Sensoren ist. Beginnend mit einer statistischen Betrachtung der Sensorcharakteristik sollen Erkenntnisse über die Ausbaufähigkeit von Halbleiter Gas Sensoren gewonnen werden. Hierbei wurde durch die Wahl des undotierten $\mathrm{SnO}_{2}$ bewusst auf ein möglichst bekanntes Material gesetzt, um die Zahl der freien Parameter so gering wie möglich zu halten.

\section{Experimentelles}

Das verwendete $\mathrm{SnO}_{2}$ wurde über einen nasschemischen Prozess aus $\mathrm{SnCl}_{4}$ hergestellt und bei $1000^{\circ} \mathrm{C}$ für acht Stunden kalziniert. Die durchschnittliche Korngrößenverteilung liegt bei $100 \mathrm{~nm}$ [4]. Die untersuchten Sensoren bestehen aus einer mittels Siebdruckverfahren auf $\mathrm{Al}_{2} \mathrm{O}_{3}$-Substrate aufgebrachter sensitiver Schicht des gewonnenen undotiertem $\mathrm{SnO}_{2}$. Die Dicke der Schicht beträgt $50 \mu \mathrm{m}$. Nach dem Siebdruck wurden die Sensoren für $24 \mathrm{~h}$ bei Raumtemperatur getrocknet und anschließend einer finalen thermischen Nachbehandlung unterzogen $\left(400-600^{\circ} \mathrm{C}\right)$. Für die durchgeführten Widerstandsmessungen enthalten die Sensoren zwischen der sensitiven Schicht und dem Keramiksubstrat eine Interdigitalstruktur mit einem Fingerabstand von $300 \mu \mathrm{m}$. Um verschiedene Arbeitstemperaturbereiche der Sensoren zu gewährleisten sind die Substrate rückseitig mit einer Heizstruktur ausgestattet. In einer abgedichteten TeflonKammer befinden sich vier $\mathrm{SnO}_{2}$-Sensoren nach oben beschriebenem Aufbau. Die Sensoren werden dabei in einem Teflon-Sockel befestigt welcher über Kontakte die Elektroden mit einem Multimeter (Keithley DMM 199) verbindet, um die Widerstandsänderungen aufzuzeichnen. Ein Netzgerät (HP 6023A) heizt die Sensoren auf die gewünschte Betriebstemperatur. Vor jeder Messung wurden die Sensoren unter trockener synthetischer Luft für mehrere Stunden stabilisiert. Als Testgase wurden Kohlenstoffmonoxid sowie Wasserstoff in niedrigen Konzentrationen in trockener und feuchter (50\% relative Feuchte) synthetischer Luft eingesetzt. 


\section{Ergebnisse und Diskussion}

In Bild 1 ist die gemittelte Widerstandmessung von insgesamt acht Sensoren (4 x Pt-Elektrode; 4 x AuElektrode) bei $250^{\circ} \mathrm{C}$ sowie $350^{\circ} \mathrm{C}$ aufgetragen.

Gezeigt wird die Antwort auf vier verschiedene aufeinanderfolgende Pulse steigender Konzentration von CO

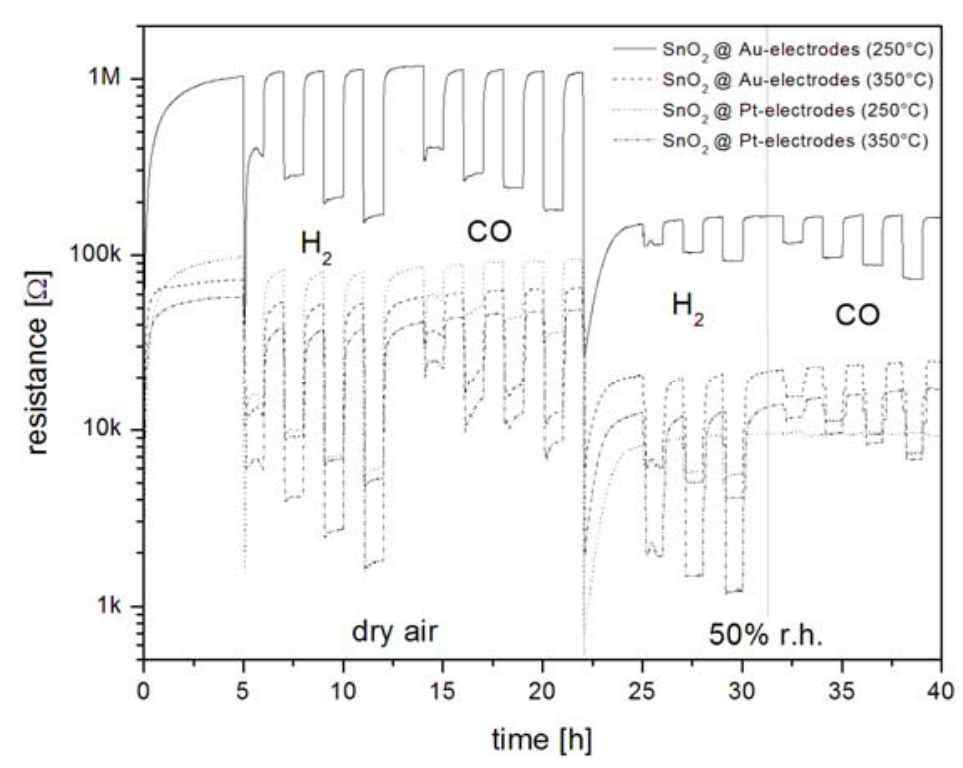

Bild 1 DC-Messung in trockener \& feuchter Luft (30-50[nur in trockener Luft]-100-200 ppm H2 \& 25-75-125-250 ppm CO und $\mathrm{H}_{2}$ in trockener und feuchter synthetischer Luft. Wie zu erwarten, zeigen sowohl die Sensoren mit Platin Elektroden als auch die Gold Elektroden einen Rückgang des Grundwiderstands $\mathrm{R}_{0}$ um die gleiche Größenordnung bei einem Anstieg von trockener Luft auf $50 \%$ relative Feuchte durch die Ausbildung von Hydroxyl-Gruppen and der Oberfläche und die dadurch verbundene Reduzierung der freien Ladungsträger. Ansprech- und Regenerationszeiten beider Sensortypen sind annähernd gleich, somit konnte bezüglich des Antwortverhaltens keine direkte Abhängigkeit des Elektrodenmaterials gefunden werden. Beide Sensortypen zeigen im Verlauf der Messung eine hohe Stabilität von $\mathrm{R}_{0}$ über die Zeit, es sind keine Drift-Phänomene zu erkennen.

Erste Tendenzen zeigen sich bezüglich der unterschiedlichen Arbeitstemperaturen der

Sensoren. Während sich die Grundwiderstände $\mathrm{R}_{0}$ bei $250^{\circ} \mathrm{C}$ für beide Materialien erheblich unterscheiden die Gold Elektroden unterstützten Sensoren zeigen hier einen um eine Größenordnung höheren Wert für $\mathrm{R}_{0}$ an - nähert sich $\mathrm{R}_{0}$ beider Typen bei $350^{\circ}$ an .

In Bild 2 sind die Grundwiderstände in trockener Luft zu Beginn jeder Messung gegen die Temperatur aufgetragen. Es zeigt zwei aufeinanderfolgende Messreihen, bei welchen jeweils eine Messung bei $250^{\circ} \mathrm{C}$ und eine weitere bei $350^{\circ} \mathrm{C}$ Arbeitstemperatur der Sensoren durchgeführt wurden. Bei den gemessenen Sensoren mit Platin-Elektroden ist eine erhöhte Temperaturstabilität des Ausgangswiderstands gegeben. Auch die Reversibilität des Ausgangwiderstands bei verschiedenen Temperaturen konnte über wiederholte Messungen bestätigt werden. Im Falle von Gold Elektroden kann der ursprüngliche Ausgangswert von $\mathrm{R}_{0}$ in einem zweiten Messzyklus nicht mehr erreicht werden. Capone et al. beschreibt eine temperaturabhängige Diffusion des Elektrodenmaterials im Falle von Gold in die sensitive Schicht [5]. Phänomene dieser Art können den Leitwert des Sensors maßgeblich beeinflussen und sind möglicherweise eine Erklärung für die geringere Stabilität und Reversibilität von $\mathrm{R}_{0}$ des Sensors mit Gold Elektroden über

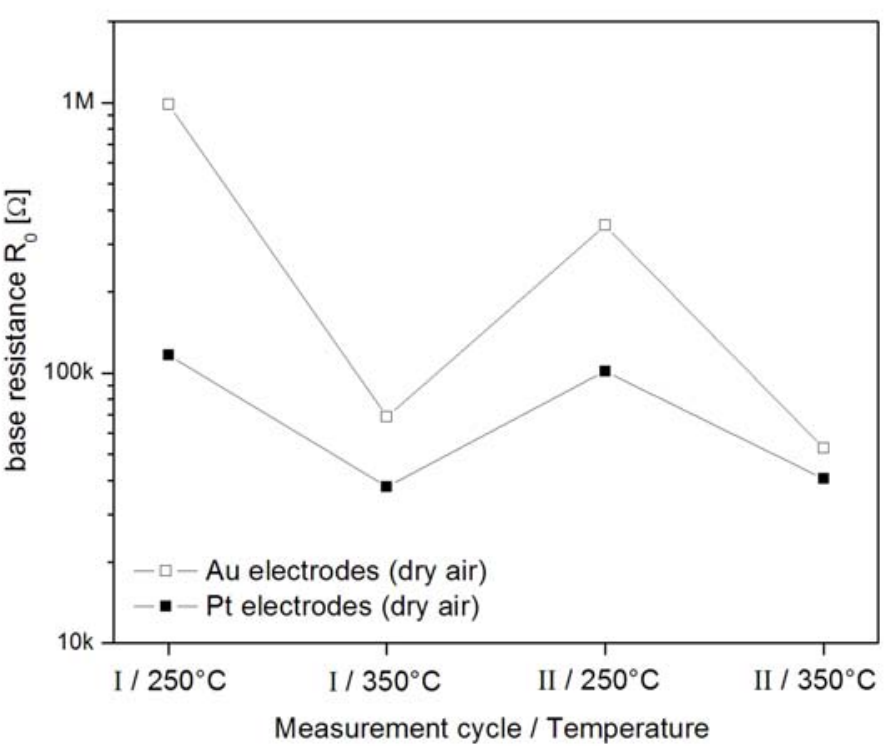

Bild 2 Grundwiderstand $\mathrm{R}_{0}$ (in trockener Luft) in Abhängigkeit zur Temperatur bestimmte Temperaturbereiche. 


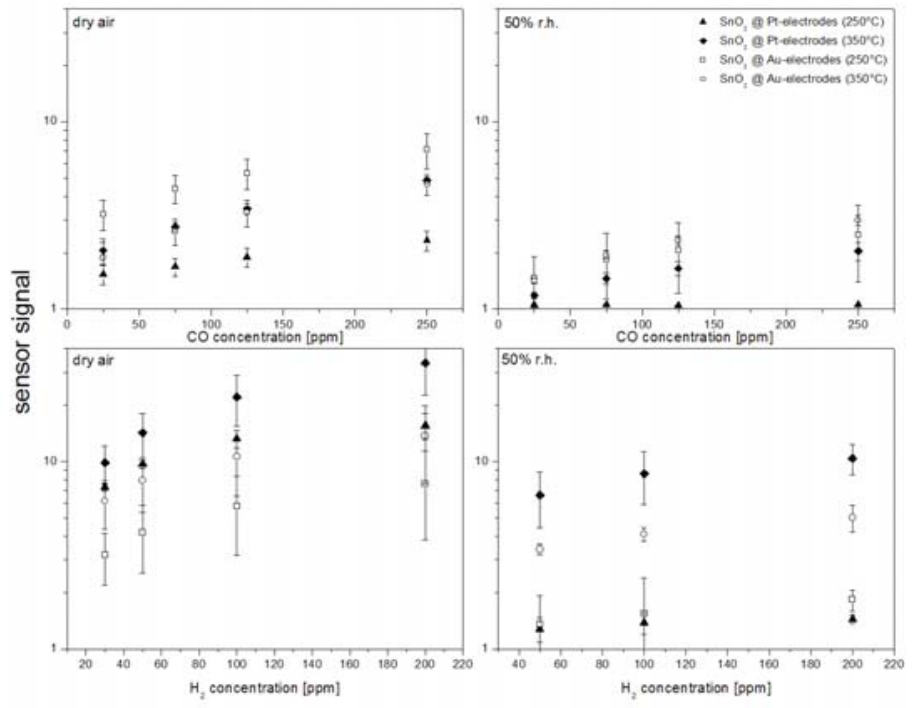

Bild 3 Sensorsignale (R0/R) gegen die Konzentration für a) CO in trockener Luft b) $\mathrm{CO}$ in 50\%r.h. c) $\mathrm{H}_{2}$ in trockener Luft d) $\mathrm{H}_{2}$ in $50 \%$ r.h.
In Bild 3 werden die Sensorsignale $\mathrm{R} / \mathrm{R}_{0}$ entsprechend ihrer Arbeitstemperatur sowie dem gewählten Elektrodenmaterial gegen die Gaskonzentration dargestellt. Für CO in trockener Luft liefern die Sensoren mit Gold Elektroden bei $250^{\circ} \mathrm{C}$ das höchste Signal, während bei $350^{\circ} \mathrm{C}$ keine Materialabhängigkeit zu erkennen ist beide Sensortypen folgen hier demselben Trend bei annähernd gleichen Werten. Betrachtet man die Werte für Kohlenmonoxid in 50\% relativer Feuchte, so zeigen Sensoren mit Platin Elektroden verschwindend geringe Signale, wohingegen Sensoren mit Gold Elektroden eine deutlich höhere Sensitivität aufzeigen, was zu einem deutlich höheren Signal führt. Bei Wasserstoff in trockener Luft geben die Sensoren mit Platin Elektroden die besten Antworten. Auch bei $50 \%$ r.h. führen die Sensoren mit Platin Elektroden zu den höchsten Signalen. Eine Anpassung nach dem Potenzgesetz $\left(y=a \cdot x^{b}\right)$ liefert eine deutliche Tendenz (Tafel 1) bezüglich der Sensitivität, wonach für die Detektion von Kohlenmonoxid Sensoren mit Gold Elektroden die besseren Parameter liefern, im Falle von Wasserstoff die Platin Elektroden unterstützten Sensoren.

Tafel 1 Anpassung des Sensorsignals (Potenz Gesetz)

\begin{tabular}{|c|c|c|}
\hline$y=a \cdot x^{b}$ & $\mathrm{a}$ & $\mathrm{b}$ \\
\hline \multicolumn{3}{|c|}{ CO (dry air) } \\
\hline Pt $250^{\circ} \mathrm{C}$ & 0,7810 & 0,1912 \\
\hline $\mathrm{Au} 250^{\circ} \mathrm{C}$ & 0,9537 & 0,3609 \\
\hline $\mathrm{Pt} 350^{\circ} \mathrm{C}$ & 0,4952 & 0,4096 \\
\hline $\mathrm{Au} 350^{\circ} \mathrm{C}$ & 0,4397 & 0,4229 \\
\hline \multicolumn{3}{|c|}{ CO (50\% r.h.) } \\
\hline Pt $250^{\circ} \mathrm{C}$ & 1,0508 & 0,0003 \\
\hline $\mathrm{Au} 250^{\circ} \mathrm{C}$ & 0,6750 & 0,2341 \\
\hline Pt $350^{\circ} \mathrm{C}$ & 0,5172 & 0,2455 \\
\hline $\mathrm{Au} 350^{\circ} \mathrm{C}$ & 0,4731 & 0,3326 \\
\hline \multicolumn{3}{|c|}{ H2 (dry air) } \\
\hline Pt $250^{\circ} \mathrm{C}$ & 2,2343 & 0,3718 \\
\hline $\mathrm{Au} 250^{\circ} \mathrm{C}$ & 0,7164 & 0,4483 \\
\hline Pt $350^{\circ} \mathrm{C}$ & 1,2344 & 0,6234 \\
\hline $\mathrm{Au} 350^{\circ} \mathrm{C}$ & 1,5720 & 0,4111 \\
\hline \multicolumn{3}{|c|}{ H2 (50\% r.h.) } \\
\hline Pt $250^{\circ} \mathrm{C}$ & 0,9265 & 0,0844 \\
\hline $\mathrm{Au} 250^{\circ} \mathrm{C}$ & 0,5676 & 0,2203 \\
\hline Pt $350^{\circ} \mathrm{C}$ & 1,9400 & 0,3187 \\
\hline $\mathrm{Au} 350^{\circ} \mathrm{C}$ & 1.1151 & 0.2841 \\
\hline
\end{tabular}

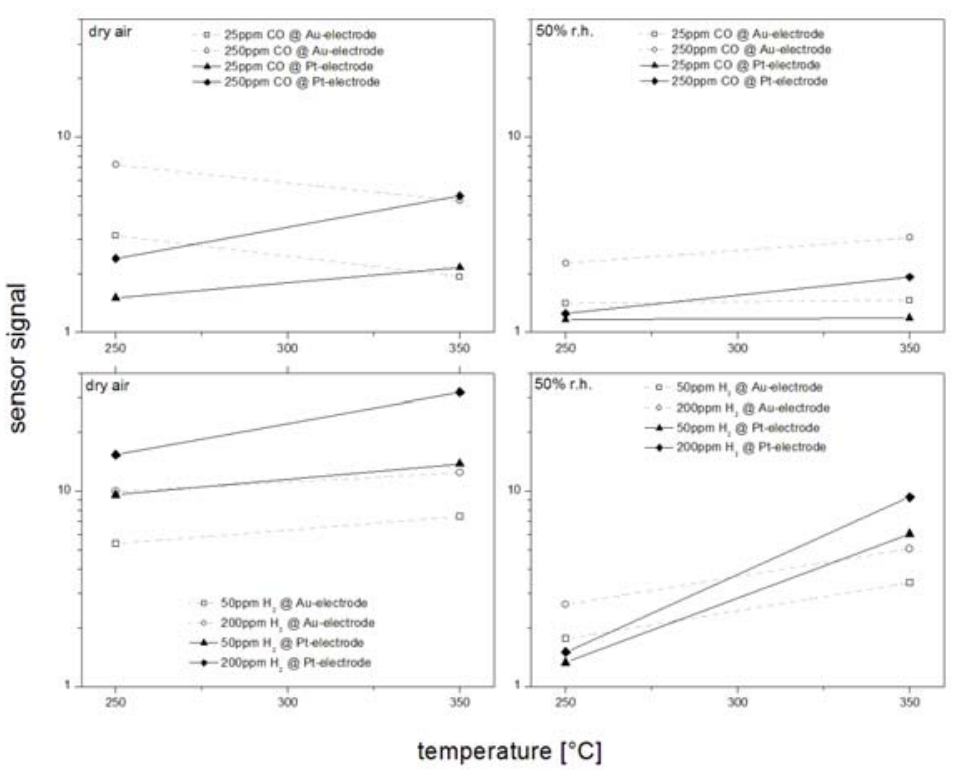

Bild 4 Sensorsignale (R0/R) gegen die Temperatur für a) CO in trockener Luft b) $\mathrm{CO}$ in 50\%r.h. c) $\mathrm{H}_{2}$ in trockener Luft d) $\mathrm{H}_{2}$ in 50\%r.h.

Setzt man das Sensorsignal in Bezug zur Arbeitstemperatur (Bild 4) ist zu erkennen, dass für Wasserstoff in trockener und feuchter Luft das Sensorsignal für jeden Elektrodentyp mit der Temperatur steigt. Für 
Kohlenmonoxid in trockener Luft nimmt das Signal bei Platin Elektroden mit der Temperatur zu, bei Gold Elektroden jedoch wurde ein abnehmendes Signal mit zunehmender Temperatur gemessen.

\section{Zusammenfassung}

Bei den gemessen $\mathrm{SnO}_{2}$ Sensoren mit verschiedenem Elektrodenmaterial wurde in erster Linie aufgezeigt, dass Platin Elektroden mehr Stabilität bezüglich Temperaturänderungen aufweisen, während Änderungen der relativen Feuchte beide Typen gleichermaßen beeinflussen. Für die Sensorsignale konnten je nach Gas Typ Präferenzen bezüglich des Elektrodenmaterials ausgemacht werden (SS[CO]: $\mathrm{Au}>\mathrm{Pt}$; SS[H2]: $\mathrm{Pt}>\mathrm{Au}$ ). Speziell für CO in trockener Luft, welches normal infinitesimale Signale für undotiertes $\mathrm{SnO}_{2} \mathrm{mit} \mathrm{Pt}$ Elektroden zeigt, kann durch eine geeignete Wahl des Elektrodenmaterials und der Temperatur die Sensoreigenschaften verbessert werden.

\section{Ausblick}

Da mit dem Elektrodenmaterial ein Parameter mit starkem Einfluss auf das Sensor Verhalten vorliegt, ist ein tieferes Verständnis dieser Beeinflussung unerlässlich um die dadurch einhergehenden Optimierungsoptionen durch einen weiteren Freiheitsgrad voll auszuschöpfen. Die statistische Betrachtung soll ein Grundverständnis liefern, um über gezielte Operando-Messungen die chemischen Aspekte zu begreifen.

\section{Literatur}

[1] S. Saukko, V. Lantto; Influence of electrode material on properties of $\mathrm{SnO}_{2}$-based gas sensor; Thin Solid Films 436 (2003); S. 137-140

[2] U. Jain, A.H. Harker, A.M. Stoneham \& D.E. Williams; Effect of electrode geometry on sensor response; Sensors and Actuators B 2 (1990); S. 111-114

[3] N. Barsan, U. Weimar; Conduction Model of Metal Oxide Gas Sensors; Journal of Electroceramics 7 (2001); S. 143-167

[4] J. Kappler; Charakterisierung von $\mathrm{SnO}_{2}$-Hochleistungsgassensoren für die CO Detektion durch in situ Methoden; Dissertation (2001); Eberhard-Karls-Universität Tübingen

[5] S. Capone, P. Siciliano, F. Quaranta, R. Rella, M. Epifani, L. Vasanelli; Moisture influence and geometry effect of $\mathrm{Au}$ and Pt electrodes on $\mathrm{CO}$ sensing response of $\mathrm{SnO}_{2}$ microsensors based on sol-gel thin film; Sensors and Actuators B 77 (2001); S. 503-511 